\title{
Solar Power Generation and Wireless Power Transmission System
}

\author{
Saurabh Deshmukh ${ }^{1}$, Ameya Kulkarni ${ }^{2}$ \\ ${ }^{I}$ (Department of Electronics and Telecommunication, Saraswati college of Engineering, University of Mumbai, \\ India) \\ ${ }^{2}$ (Department of Electronics, Datta Meghe College of Engineering, University of Mumbai, India)
}

\begin{abstract}
In the age of wireless technology and increasing use of renewable energy there is a constant increase in the demand for wireless technology which is environment friendly. The first step in wireless power is providing power to any electronic device wirelessly. If the efficiency of transmitting the power wirelessly is increased slightly further, then wireless power transmission could become a standard means for charging any electronic gadget, and also if it is by the means of a renewable and a clean power source such as Solar energy it would be an cherry on top of the cake.
\end{abstract}

Keywords: MOSFET/IGBT: Metal Oxide semiconductor field effect transistor/Insulated gate bipolar junction transistor, Resonance: resonance is the tendency of a system to oscillate with greater amplitude at some frequencies than at others, Wavelength: This distance as a distinctive feature of radio waves from a transmitter, WiTricity: It's an abbreviation used for the new upcoming technology "Wireless Electricity", Solar irradiance is a measure of the irradiance (power per unit area on the Earth's surface) produced by the Sun in the form of electromagnetic radiation.

\section{Introduction}

Transmission of electrical energy without the use of wires that depends upon electrical conductivity was first introduced by Sir Nikola Tesla in the year 1891. Witricity, an abbreviation of wireless transfer of electricity, is a term introduced initially by Dave Gerding in the year 2005.This principle of wireless electricity transfer works on the principle of using coupled resonant objects for the transferring electricity. Wireless energy transfer can be useful in such applications as providing power to autonomous electrical and electronic devices. This energy which is transferred can be derived from a renewable source; the best available option is the Solar Energy.

Solar energy is harnessed by the means of Solar Cells. The solar cells are made of silicon crystals that have been combined with other materials in such a way that there are extra electrons in one part of the cell, and missing electrons in another part of the cell. When the sunlight strikes the cell, photons in the light knock some of the extra electrons loose from the silicon, and they flow to the part of the cell that is missing electrons. This flow produces an electrical current that eventually reaches the inverter, where it gets converted into usable electricity.

The overall goal of this paper is to design and implement a clean power generation and a wireless power transmission system.

\section{Materials And Methods}

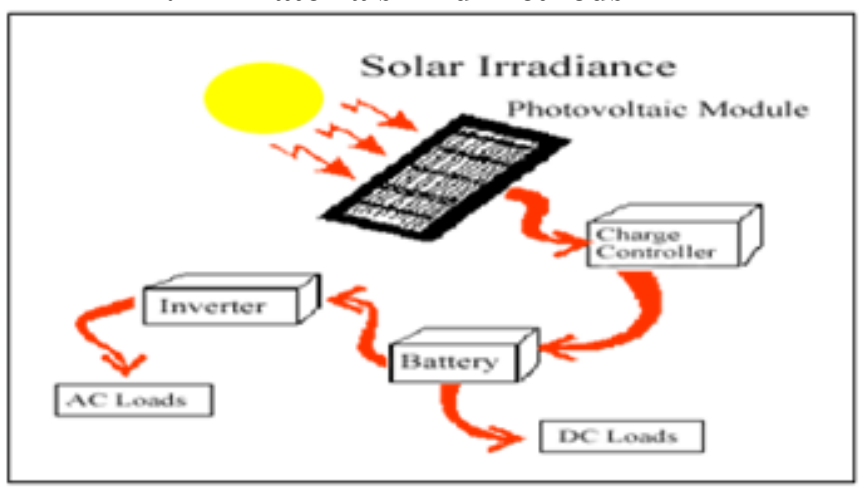

The Photovoltaic module (PV) or Solar panels are installed on the roofs, they convert the sunlight into the direct current(DC) power. The charge controller limits the rate at which the electric current is added or drawn from the electric batteries. The batteries being one of the main component of the solar power system, the 
charge controller protects the batteries from overcharging and also against overvoltage. This surely does increase the life span of batteries. The DC power from the solar panel is sent to the inverter where it is converted into Alternating current (AC) power.

Solar Inverter -

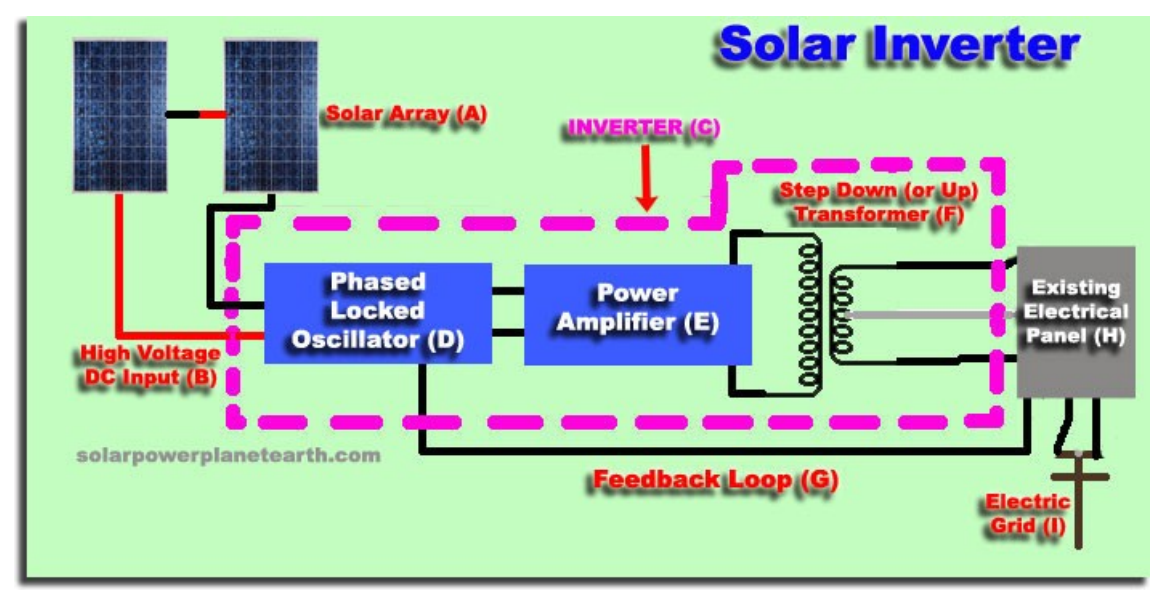

The block diagram schematic of the Solar inverter clearly shows us that it implements a Phase Locked Loop oscillator wit a Power Amplifier and finally a step up/down transformer connected to the out section .The basic function of a Phase Locked Loop oscillator is ,it generates a output signal whose phase is related to the phase of the input signal. The oscillator's function is to generate a periodic signal. The phase detector compares the phase of that signal with the phase of the input periodic signal and adjusts the oscillator to keep the phases matched. The power amplifier as the name suggests is used for a greater level of amplification of the signal. The transformer which is connected to the out section of the amplifier can be used for stepping up or stepping down the signal, which can done according the application. This Alternating current is then made to flow in the AC line. The power from these AC lines is then transferred wirelessly for powering the domestic devices. Here the principle of witricity comes into picture. Witricity is based on strong coupling between electromagnetic resonant objects to transfer energy wirelessly between them. This differs from other methods like simple induction, microwaves, or air ionization. The system consists of transmitters and receivers that contain magnetic loop antennas critically tuned to the same frequency. Due to operating in the electromagnetic near field, the receiving devices must be no more than about a quarter wavelengths from the transmitter. Unlike the far field wireless power transmission systems based on travelling electro-magnetic waves, Witricity employs near field inductive coupling through magnetic fields similar to those found in transformers except that the primary coil and secondary winding are physically separated, and tuned to resonate to increase their magnetic coupling. These tuned magnetic fields generated by the primary coil can be arranged to interact vigorously with matched secondary windings in distant equipment but far more weakly with any surrounding objects or materials such as radio signals or biological tissue.

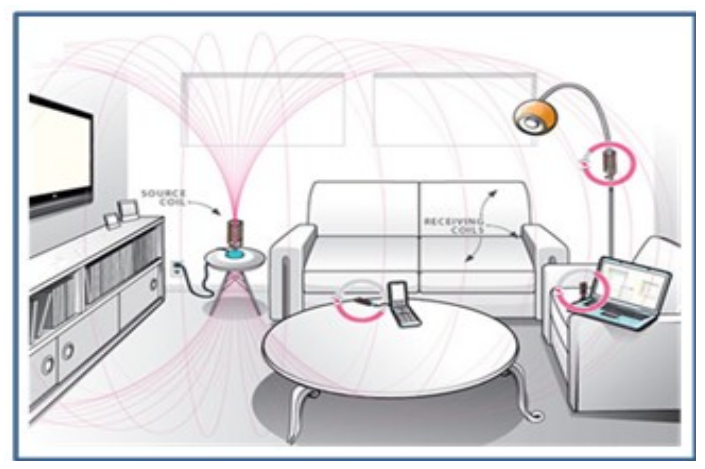

\section{STEP 1:-}

III. How It Works?

A circuit $[\mathrm{A}]$ attached to the wall socket converts the standard 60-hertz current to 10 megahertz and feeds it to the transmitting coil [B]. The oscillating current inside the transmitting coil causes the coil to emit a 10-megahertz magnetic field. 


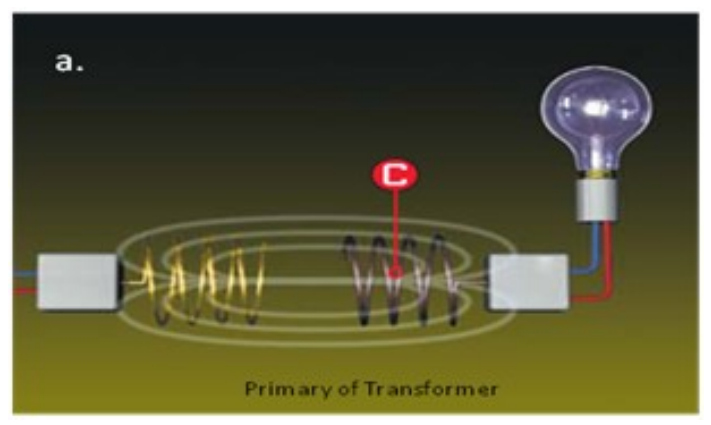

STEP 2:-

The receiving coil $[\mathrm{C}]$ has the exact same dimensions as the sending coil and thus resonates at the same frequency and, in a process called magnetic induction, picks up the energy of the first coil's magnetic field.

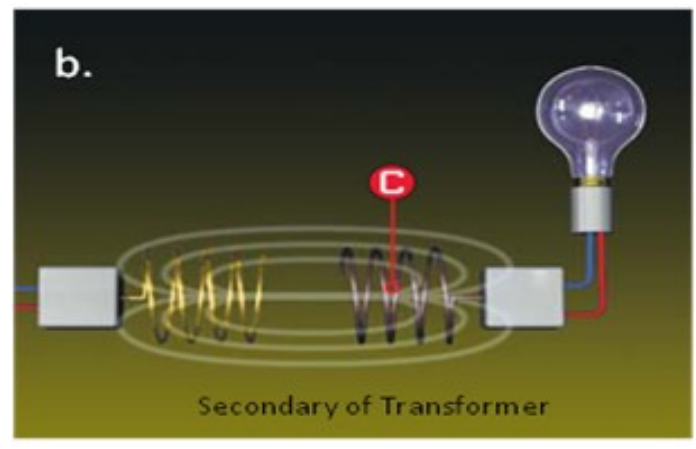

STEP 3:-

The energy of the oscillating magnetic field induces an electrical current in the receiving coil, lighting the bulb [D].

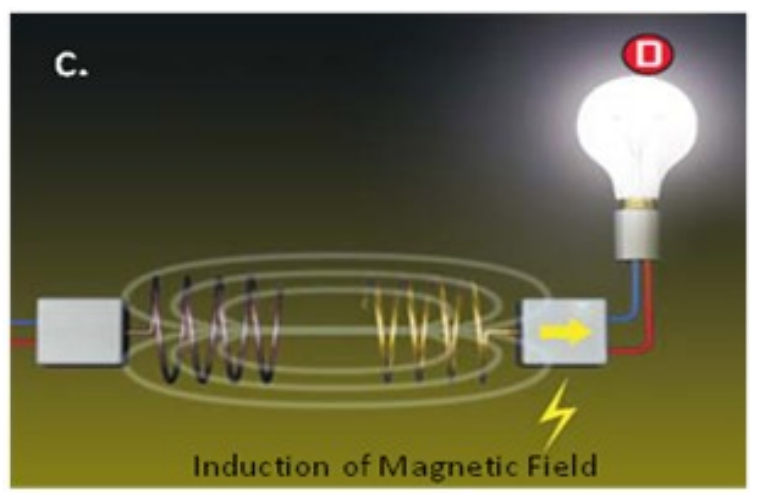

\section{Block Diagram}

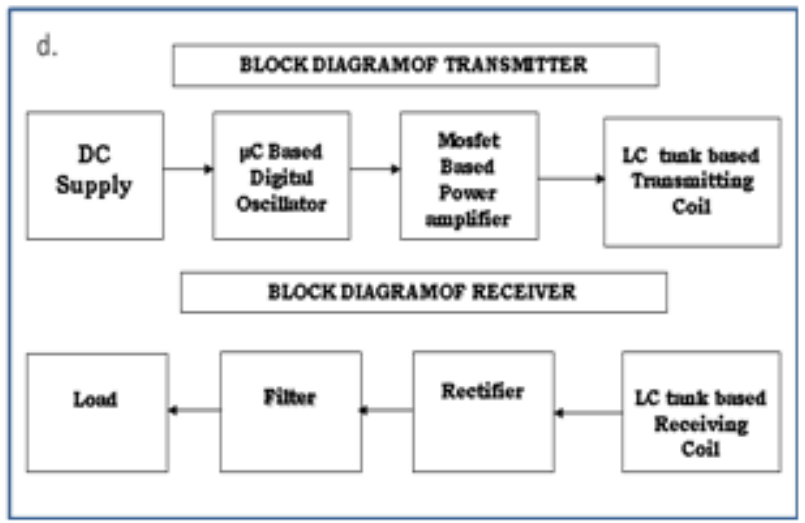




\section{Working of Transmitter}

The input from mains is given to the power and frequency controller. The output of this system is given to MOSFET/IGBT.

The main purpose of using MOSFET/ IGBT is to convert DC to AC and also for amplifying square wave at the gate input. The voltage given to the transmitting coil generates magnetic field around it. The capacitor is connected to the coil parallel and hence the resonating circuit is formed.

Until the resonant frequency of receiving coil matches with the resonant frequency of the transmitting coil magnetic field won't get induced in the receiving coil. For this purpose of matching the resonant frequency we used different values of " $\mathrm{L}$ " and "C" for resonant frequency matching purpose.

To match the resonant frequency of the receiver and the transmitter coil we used the switches to vary the time periods of the square wave by which we are controlling the frequency at output.

\section{Working of Receiver}

As the receiving coil comes in the range of the magnetic field of the transmitting coil, the voltage across the transmitting coil gets induced in the receiving coil because of mutual inductance and matching of resonance frequency The received voltage is in AC form, we have to convert it into DC for DC load hence we used a rectifier circuit which provides constant DC at the output for driving the load. And if the load is ac load then we can give direct output to it.

\section{Calculation}

The transmitter is supplied with 18 volts AC and the current circulating in it will induce an electromotive force in the receiving coil that will drive the light bulb. The electromagnetic wave is used to radiate energy with its oscillating electric and magnetic fields. To make sure the field stays strong, the two sides will be made to resonate at the same magnetic frequency of $40 \mathrm{kHz}$.

For calculating the resonant frequency we used the following formula:

Where,

$\mathrm{F}=$ resonant frequency.

$\mathrm{L}=$ inductance value of coil.

$\mathrm{C}=$ capacitance value of coil.

\section{Formula For Finding Inductance}

$$
L=\frac{\mathrm{N}^{2} \mu \mathrm{A}}{1}
$$

Where,

$\mathrm{L}=$ Inductance of Coil in Henrys

$\mathrm{N}=$ Number of turns in Coil

$\mu=$ Permeability of air (core material)

$\mathrm{A}=$ Area of coil in square meters

$1=$ Average length of coil in meters

Efficiency: During the transferring of energy there are some of the losses which takes place which reduces the efficiency .There are hysteresis losses, eddy current losses, attenuation, etc. which are responsible for the same.

Less Range: As witricity is under the research sector, the range of wireless transferring of energy is still not upto the mark.

\section{Solar efficiency-}

Solar efficiency refers to the amount of light that can be converted into usable electricity.

Solar efficiency is dependent on 2 parameters-

Solar cell efficiency-Solar cell efficiency is the amount of light that the individual solar cell coverts into electricity. It is the ratio of the electrical output of the solar cell to the incident energy in the form of sunlight. This is calculated by dividing a cell's power output (in watts) at its maximum power point $\left(\mathrm{P}_{\mathrm{m}}\right)$ by input light $\left(E\right.$, in $\left.W / \mathrm{m}^{2}\right)$ and the surface area of the solar cell $\left(\mathrm{A}_{\mathrm{c}}\right.$ in $\left.\mathrm{m}^{2}\right)$. 


$$
\eta=\frac{P_{m}}{E \times A_{c}}
$$

Solar panel efficiency: - Solar panel efficiency is the amount of light that the entire module converts into electricity. The efficiency of solar panel is less than that of solar cell, due to the spacing which is present in between the cells and because of the glass covering over the panel which reflects some of the sunlight.

\section{How to improve the efficiency of the solar panel:-}

Increasing the amount of electric current produced by the solar panel can be done by augmenting its light surface with aluminum nanostructures.

Another way to increase the electric current produced by solar panel is by studying the light receiving surface of Gallium Arsenide (GaAs) devices with aluminum nano cylinders.

This magnified the scattering of light in the visible part of the spectrum which dominates the energy in sunlight, the scattered light then travels a longer path so that more photons can be absorbed and converted into current.

\section{Conclusion}

The main aim of this paper is to provide a sustainable solution to the problem of usage of the nonrenewable sources of energy. The solar energy which is an abundant form of energy can be put to maximum use provided the techniques for harnessing it should be appropriate. Witricity on the other hand provides us with a wireless solution, which is not widely implemented because of the concerns of the lack of efficiency. One of the major constraints for implementing both the technologies together would be the lack of efficiency .Our paper has mainly focused on combining both the technologies together, as this would be a major advancement in the field of technology of using a renewable and a wireless technology together. If we can overcome these constraints, then we could implement this technology in various applications such as airports, home appliances, office environment and any other public spaces.

\section{References}

[1] H. Lee, "SEEMLESS: Distributed Algorithm for Topology Control of Survivable Energy Efficient Multihop Wireless Sensor Networks Using Adjustable Transmission Power," Proc. Sixth ACIS International Conference on Software Engineering, Artificial Intelligence, Networking, and Parallel/Distributed Computing (SNPD’05), Towson, Maryland, May 2005

[2] Wenzhen Fu, Bo Zhang, Dongyuan Qiu, "Study on Frequency-tracking Wireless Power Transfer System by Resonant Coupling," Power Electronics and Motion Control Conference, pp. 2658-2663, May 2009.

[3] Wenzhen Fu, Bo Zhang, Dongyuan Qiu, "Study on Frequency-tracking Wireless Power Transfer System by Resonant Coupling," Power Electronics and Motion Control Conference, pp. 2658-2663, May 2009.

[4] Hassan, Marwa M. Framework for active solar collection systems. Tese PhD, Department of Building Construction: Virginia Polytechnic Institute and State University Blacksburg, 2003, Blacksburg, Virginia.

[5] Duffie, J. A., Beckman W., Solar Engineering of Thermal Processes, John Wiley \& Sons, Inc., edicao, 1991.

[6] Martins, Fernando Ramos, Pereira, Enio Bueno and Echer, Mariza Pereira de Souza. Solar energy resources assesment using geostationary satellites in Brazil: Swera Project. Rev. Bras. Ens. Fis., 2004, vol.26, no.2, p.145-159. ISSN 0102-4744.

[7] Albert Esser and Hans-Christoph Skudelny, "A New Approach to Power Supplies for Robots", IEEE Transactions On Industry Applications, VOL. 21, NO. 5, SEPTEMBER 1991

[8] Alanson P. Sample, David A. Meyer,and Joshua R. Smith, "Analysis, Experimental Results, and Range Adaptation of Magnetically Coupled Resonators for Wireless Power Transfer", IEEE Transactions On Industrial Electronics, VOL. 58, NO. 2, FEBRUARY 2011.

[9] Q. Yuan, Q. Chen, L. Li, and K. Sawaya, "Numerical analysis on transmis-sion efficiency of evanescent resonant coupling wireless power transfer system," IEEE Trans. Antennas Propag., vol. 58, no. 5, pp. 1751-1758,May 2010.

[10] Suja, S., Sathish Kumar, T., Dept. of EEE, Coimbatore Inst. of Technol., Coimbatore, India "Solar based wireless power transfer system" Published in Computation of Power, Energy, Information and Communication (ICCPEIC), 2013 International Conference on 17 April 2013. 